\title{
THE CON JUGACY FUNCTION
}

\author{
WALTER LEIGHTON ${ }^{1}$
}

Abstract. The conjugacy function $\delta(x)$ of the differential equation $y^{\prime \prime}+p(x) y=0$ is defined as the distance from a point $x$ to its first conjugate point. Conditions that $\delta(x)$ be convex or concave are given, as well as conditions that $\delta(x)$ be an increasing or decreasing function. The lemma provides a novel type of comparison theorem.

Consider the second-order linear differential equation

$$
y^{\prime \prime}+p(x) y=0,
$$

where $p(x)$ is positive and of class $C^{\prime \prime}$ on an interval $I:[a, \infty)$. We shall assume that solutions of (1) are oscillatory on $I$.

In this paper we study what we term the conjugacy function associated with (1). The conjugacy function $\delta(x)$ is defined as the distance from a point $x$ to its first conjugate point larger than $x$. With each point $x_{0}$ of $I$ we also associate an $f$-point. If $y(x)$ is a nonnull solution of (1) with the property that

$$
y^{\prime}\left(x_{0}\right)=0,
$$

the $f$-point of $x_{0}$ is the first point following $x_{0}$ at which $y^{\prime}(x)$ again vanishes.

It will be convenient to write

$$
p(x)=1 / h^{2}(x)
$$

We have, then, the following result.

THEOREM. If on I

(a) $p(x) \downarrow$, then $\delta(x) \uparrow$;

(b) $p(x) \uparrow$, then $\delta(x) \downarrow$;

(c) $h^{\prime \prime}(x)<0$, then $\delta(x)$ is concave;

(d) $h^{\prime \prime}(x)>0$, then $\delta(x)$ is convex.

We observe, first, that in (a) and (b) the conclusion that $\delta(x)$ is increasing or decreasing follows at once from an easy modification of

Received by the editors June 12, 1969.

AMS Subject Classification. Primary 3442.

Key Words and Phrases. Second-order linear differential equation, conjugate point: conjugacy function, comparison theorem.

1 This will acknowledge the partial support of the author by the U. S. Army Research Office (Durham) under Grant numbered DA ARO D31-124-G1007. 
a proof in $[2$, p. 3$]$. The concavity and convexity conclusions appear to require greater subtlety. We commence with a novel type of comparison theorem that we state as a lemma.

Lemma. Let $x=c$ be the first conjugate point and $x=f$ be the $f$-point of $x=x_{0}$. If $h^{\prime \prime}(x)<0$ on $I$, then $f<c$, while if $h^{\prime \prime}(x)>0$ on $I, c<f$. If $h^{\prime \prime}(x) \equiv 0, f=c$.

To prove this recall that if $y(x)$ is a solution of (1), its derivative satisfies the differential equation

$$
\left(h^{2} z^{\prime}\right)^{\prime}+z=0 .
$$

Under the transformation $z=w / h$ equation (3) becomes

$$
w^{\prime \prime}+\left(\frac{1}{h^{2}}-\frac{h^{\prime \prime}}{h}\right) w=0 .
$$

An appeal to the Sturm comparison theorem completes the proof.

The following nonoscillation result is an immediate consequence of (4) (cf. [1], [3]).

Corollary. If

$$
1-h h^{\prime \prime} \leqq 0 \quad(x \in I),
$$

the solutions of (1) are nonoscillatory.

For, if the solutions of (1) oscillate on $I$, so must the solutions of (4).

We return to the proofs of parts (c) and (d) of the theorem.

For both these cases we recall from an earlier paper [4] that if $v(x)$ is any nonnull solution of $(1), \delta\left(x_{0}\right)=c-x_{0}, d c / d x_{0}=v^{2}(c) / v^{2}\left(x_{0}\right)$, and

$$
\delta^{\prime}\left(x_{0}\right)=v^{2}(c) / v^{2}\left(x_{0}\right)-1 .
$$

We compute

$$
\delta^{\prime \prime}\left(x_{0}\right)=2 \frac{v^{2}(c)}{v^{2}\left(x_{0}\right)}\left[\frac{v(c) v^{\prime}(c)-v\left(x_{0}\right) v^{\prime}\left(x_{0}\right)}{v^{2}\left(x_{0}\right)}\right] .
$$

It will be sufficient to prove that the numerator inside the brackets is negative when (c) holds and is positive when (d) holds. Recall that $v(x)$ was an arbitrary nonnull solution of (1), and let $x_{0}$ be any point of $I$. We choose $v(x)$ to be a solution the derivative of which vanishes at $x=x_{0}$. Then $v\left(x_{0}\right) \neq 0$, and, according to the lemma, the $f$-point of 
$x_{0}$ precedes the conjugate point when $h^{\prime \prime}<0$ and follows it when $h^{\prime \prime}>0$. It follows that the numerator inside the brackets in (6) is negative when condition (c) holds and positive when condition (d) holds-as reference to a figure will make quite plain.

The proof of the theorem is complete. ${ }^{2}$

An application. Consider the functional

$$
J=\int_{a}^{b}\left[y^{\prime 2}-p(x) y^{2}\right] d x
$$

where $p(x)$ is positive and of class $C^{\prime \prime}$ on a suitable interval $I$. According to the customary integration by parts

$$
J=\left.y y^{\prime}\right|_{a} ^{b}-\int_{a}^{b} y\left[y^{\prime \prime}+p(x) y\right] d x
$$

Thus, if $x=b$ is a conjugate point of $x=a$, and if $y(x)$ is a solution of (1) that vanishes at $x=a$, it will follow that $J=0$. Note that along any solution $v(x)$ of (1),

$$
J=v(b) v^{\prime}(b)-v(a) v^{\prime}(a) .
$$

From (6), if $x=b$ is conjugate to $x=a$ and $v(a) \neq 0$, the sign of $J$ will be negative, if $h^{\prime \prime}(x)<0$ on $[a, b]$ and positive, if $h^{\prime \prime}>0$ on the interval. When $h^{\prime \prime}(x) \equiv 0$ on $[a, b], J=0$.

\section{REFERENCES}

1. R. L. Potter, On self-adjoint differential equations of second order, Pacific J. Math. 3 (1953), 467-491. MR 15, 32.

2. A. Samanich Skidmore and Walter Leighton, On the oscillation of solutions of a second-order linear differential equation, Rend. Circ. Mat. Palermo (2) 14 (1965), 327-334. MR 35 \#5706.

2 The referee has directed the writer's attention to the recent interesting book by O. Borůvka, Lineare differentialtransformationen 2. Ordung (VEB Deutscher Verlag der Wissenschaften, Berlin (1967)). In this treatise the author provides much valuable material including what amounts to a proof for parts (a) and (b) of the present theorem that is an interesting alternative to the method used in [2]. The formula for $d c / d x_{0}$ preceding equation (6), which (with some other similar results) appears to have been first observed in [4] has been rediscovered by Borůvka (p. 113). He also provides other results of this kind (some of which do not appear in [4]). 
3. Walter Leighton, On self-adjoint differential equations of second order, J. London Math. Soc. 27 (1952), 37-47. MR 13, 745.

4. - Principal quadratic functionals, Trans. Amer. Math. Soc. 68 (1949), 253-274. MR 11, 603.

5. Walter Leighton and A. D. Martin, Quadratic functionals with a singular end point, Trans. Amer. Math. Soc. 78 (1955), 98-128. MR 16, 598.

UNIVERSITY OF MISSOURI 